\title{
The impact of the circumstellar magnetic field on the resulting gamma-ray emission from supernova remnants
}

\author{
lurii Sushch* \\ DESY, 15738 Zeuthen, Germany \\ Centre for Space Research, North-West University, 2520 Potcheftroom, South Africa \\ Astronomical Observatory of Ivan Franko National University of L'viv, vul. Kyryla i Methodia, \\ 8, L'viv 79005, Ukraine \\ E-mail: iurii.sushch@desy.de
}

\section{Robert Brose}

DESY, 15738 Zeuthen, Germany

Institute of Physics and Astronomy, University of Potsdam, 14476 Potsdam, Germany

\section{Martin Pohl}

DESY, 15738 Zeuthen, Germany

Institute of Physics and Astronomy, University of Potsdam, 14476 Potsdam, Germany

\begin{abstract}
Supernova remnants (SNRs) are widely believed to be one of the main candidates for the origin of Galactic cosmic rays. The observed very-high-energy gamma-ray emission observed from a number of SNRs suggests that particles are indeed accelerated to high energies at the shock of the remnants. However, it is extremely difficult to determine which particles are responsible for this emission as both protons (through hadronic interactions and subsequent pion decay) and electrons (through inverse Compton scattering on ambient photon fields) can potentially generate gammaray photons. Recent detection of the abrupt cut-off at lower energies in the gamma-ray spectra of two SNRs, IC 443 and W44, with the Fermi-LAT provided a strong evidence that cosmic-ray protons are indeed accelerated in SNRs based on the interpretation of this cutoff as a characteristic pion-decay feature. However, it cannot be firmly excluded that a similar spectral feature can be formed also in the leptonic scenario as a result of some spatial or temporal variability of the ambient medium. SNRs created in core-collapse explosions expand inside the stellar wind bubble blown up by a progenitor star. The magnetic field in the wind medium follows a $1 / \mathrm{r}$ profile with high values at the surface of the star, e.g. 1-10 G for red supergiants. This means that at early stages of its evolution the remnant interacts with a very strong magnetic field, which results in a synchrotron cooling feature in the electron spectrum, which in turn shows up in the gamma-ray spectrum as a break at similar energies as a pion-decay signature.
\end{abstract}

36th International Cosmic Ray Conference -ICRC2019-

July 24th - August 1st, 2019

Madison, WI, U.S.A.

* Speaker. 


\section{Introduction}

Supernova remnants (SNRs) are widely considered to be the main candidates for the acceleration sites of Galactic cosmic rays (CRs), which places them among the most interesting and most studied astrophysical objects. The very-high-energy gamma-ray emission observed from SNRs implies that particles are indeed accelerated to high energies at the shocks of the remnants. This emission, however, can be explained by both electrons (through inverse Compton scattering on ambient photon fields) and protons (through hadronic interactions with subsequent decay of neutral pions) and it is very difficult to discriminate between these two scenarios. Although acceleration of electrons in SNRs can be directly confirmed through the detection of synchrotron emission in the X-ray energy band, similar confirmation for protons would require a careful analysis of the observed gamma-ray emission. Recently, for several old SNRs, such as IC 443 and W44, the abrupt cut-off was detected in the gamma-ray spectrum at $\sim 0.1 \mathrm{GeV}$, which was interpreted as a characteristic pion-decay feature [1]. This detection provides a strong direct evidence that protons are indeed accelerated at the SNRs shocks as such a cut-off is usually not expected in the leptonic scenario.

Majority of SNRs in the Galaxy are produced in core-collapse events. In such a case a remnant is evolving inside a stellar wind bubble blown up by a progenitor star. Magnetic field in the circumstellar environment follows a $1 / r$ dependance on the radial distance, which means that at early stages of its evolution an SNR would interact with a very high magnetic field. This field would naturally result in severe synchrotron losses which may considerably modify the resulting electron spectrum and thus the expected gamma-ray spectrum generated by electrons. In this scenario synchrotron losses might cause a cooling break in the spectrum appearing at quite low $\mathrm{GeV}$ energies resembling a characteristic pion-decay feature. In this work we aim to study such a possibility through the simulation of the SNR evolution and particle acceleration in the circumstellar environment of the stellar wind bubble of the progenitor star using the RATPaC (Radiation Acceleration Transport Parallel Code) code [2, 3, 4, 5].

\section{Modeling}

\subsection{Hydrodynamics}

The standard gasdynamical equations

$$
\frac{\partial}{\partial t}\left(\begin{array}{c}
\rho \\
\vec{m} \\
E
\end{array}\right)+\nabla\left(\begin{array}{c}
\rho \vec{v} \\
\overrightarrow{m v}+P \vec{I} \\
(E+p) \vec{v}
\end{array}\right)^{T}=\left(\begin{array}{l}
0 \\
0 \\
0
\end{array}\right), \quad \frac{\rho \vec{v}^{2}}{2}+\frac{P}{\gamma-1}=E
$$

are solved, where $\rho$ is the density of the thermal gas, $\vec{v}$ the plasma velocity, $\vec{m}=\vec{v} \rho$ the momentum density, $P$ the thermal pressure of the gas, $\vec{I}$ the unit tensor, and $E$ the total energy of the ideal gas with $\gamma=5 / 3$. We assume that the magnetic field is dynamically unimportant due to its low strength and the remnant not being in the radiative phase yet [6]. The equations are solved in 1D for a spherical symmetry.

We initialize the ejecta profile by a plateau in density with the value $\rho_{\mathrm{c}}$ up to the radius $r_{\mathrm{c}}$ followed by a power-law distribution up to the ejecta-radius $R_{\mathrm{ej}}$ : 


$$
\rho(r)= \begin{cases}\rho_{\mathrm{c}}, & r<r_{\mathrm{c}}, \\ \rho_{\mathrm{c}}\left(\frac{r}{r_{\mathrm{c}}}\right)^{-n}, & r_{\mathrm{c}} \leq r \leq R_{\mathrm{ej}}, \\ \frac{M_{\star}}{4 \pi r^{2} v_{\text {wind }}}, & r>R_{\mathrm{ej}} .\end{cases}
$$

The exponent for the ejecta profile is set to $n=9$ for the core-collapse explosion. The velocity of the ejecta is defined as

$$
v_{\mathrm{ej}}(r)=\frac{r}{T_{\mathrm{SN}}},
$$

where $T_{\mathrm{SN}}=1 \mathrm{yr}$ is the initial time set for hydrodynamic simulations. Then for the assumed mass, $M_{\mathrm{ej}}$ (see Section 3), and energy, $E_{\mathrm{ej}}$ (assumed to be $10^{51} \mathrm{erg}$ for all the cases), of the ejecta, and defining the radius of the ejecta as multiple of $r_{\mathrm{c}}, R_{\mathrm{ej}}=x r_{\mathrm{c}}$, the initial conditions for simulations can be written as

$$
\begin{aligned}
& r_{c}=\left[\frac{10}{3} \frac{E_{e j}}{M_{e j}}\left(\frac{n-5}{n-3}\right)\left(\frac{1-\frac{3}{n} x^{3-n}}{1-\frac{5}{n} x^{5-n}}\right)\right]^{1 / 2} T_{S N}, \\
& \rho_{c}=\frac{M_{e j}}{4 \pi r_{c}^{3}} \frac{3(n-3)}{n}\left(1-\frac{3}{n} x^{3-n}\right)^{-1}, \\
& v_{\mathrm{c}}=\frac{r_{\mathrm{c}}}{T_{\mathrm{SN}}}
\end{aligned}
$$

The initial temperature is set to $10^{4} \mathrm{~K}$ everywhere and the initial pressure is calculated using the equation of state. We used 262141 linearly distributed grid-points and $15 \mathrm{pc}$ grid-size for the hydro simulations.

We further assume in Eq. 2.2 that the SNR is expanding into a wind zone, i.e. the density radial distribution follows $n \propto r^{-2}$. The density in the wind zone is determined by the mass-loss rate, $\dot{M}_{\star}$, and wind velocity, $v_{\text {wind }}$, of the progenitor star. The size of the stellar wind bubble is assumed to be large enough, so that SNR expands only into the wind zone during the considered period of evolution.

\subsection{Magnetic field}

We assume that the magnetic field in the wind zone of the progenitor star, $B_{\text {wind }}$, follows a $1 / r$ dependence on the radial distance. Thus, the radial distribution of the magnetic field strength can be expressed as

$$
B_{\text {wind }}(r)=B_{*} \frac{R_{*}}{r},
$$

where $B_{*}$ is the surface magnetic field of the star and $R_{*}$ is the radius of the star.

In this study we account for the compression of the magnetic field at the shock but ignore amplification upstream of the shock. The immediate downstream magnetic field is given then by $B_{\mathrm{d}}=\sqrt{11} B_{\text {wind }}$ for the shock compression ratio of 4 . We then assume that the immediate downstream magnetic field is transported inside the SNR with the plasma flow and evolves following the induction equation for ideal MHD. 


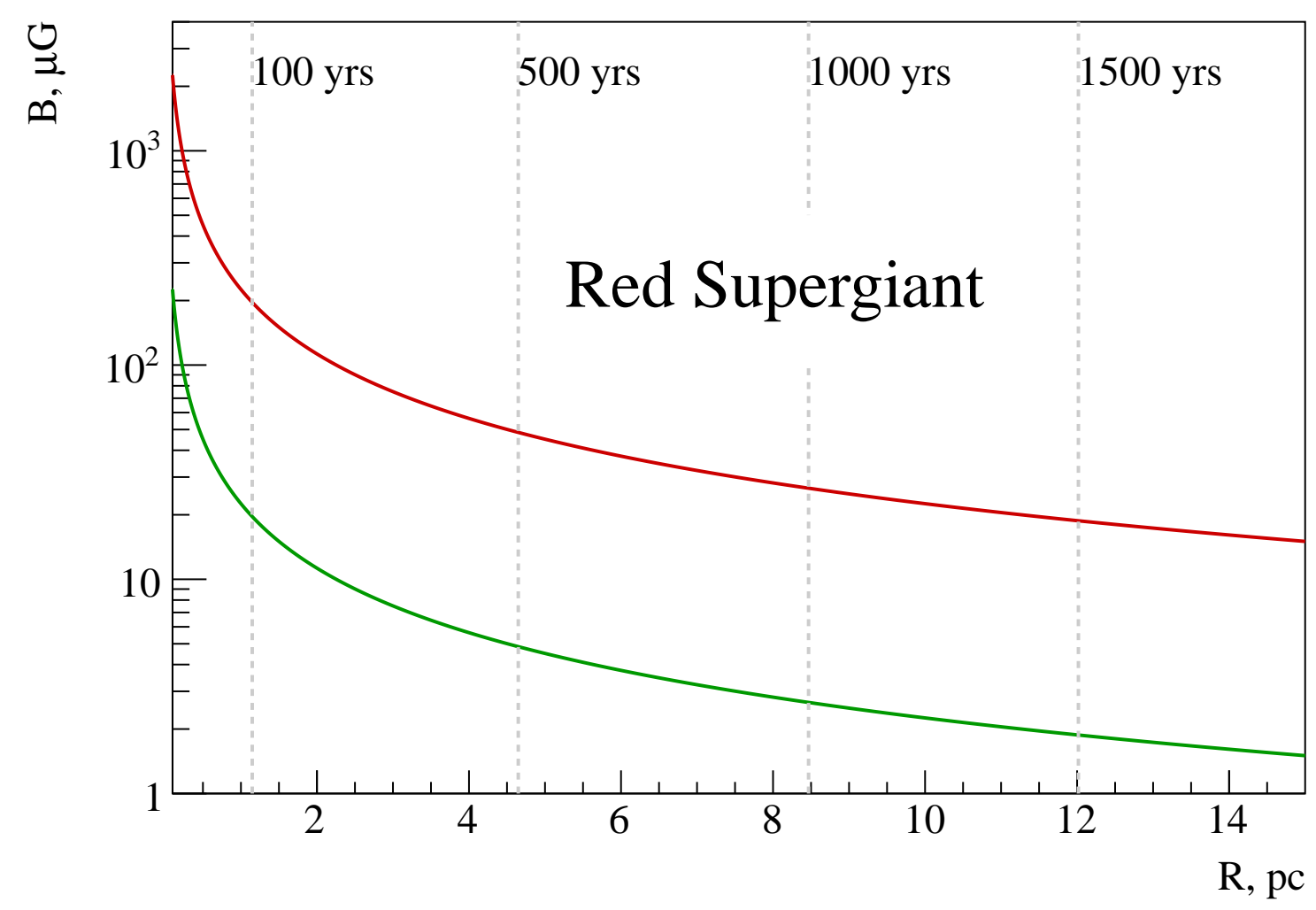

Figure 1: Radial distribution of the circumstellar magnetic field strength in the stellar wind bubble of the RSG for two cases described in the Section 3: cool scenario (red line) and not cool scenario (green line). Grey dashed vertical line correspond to the size of the remnant at a given age.

\subsection{Particle acceleration}

We simulate the particle density evolution solving the transport equation in the form

$$
\frac{\partial N}{\partial t}=\nabla(D \nabla N-\vec{v} N)-\frac{\partial}{\partial p}\left((N \dot{p})-\frac{\nabla \vec{v}}{3} N p\right)+Q
$$

where $N$ is the differential number density of cosmic rays, $D$ is the spatial diffusion coefficient (assumed to be Bohm-like), $\vec{v}$ is the plasma velocity, $\dot{p}$ represents the energy losses (in our case synchrotron losses) and $Q$ is the source term.

Particle injection is determined by the source term which is given by

$$
Q=\eta n_{\mathrm{u}}\left(V_{\mathrm{s} h}-V_{\mathrm{wind}}\right) \delta\left(R-R_{\mathrm{s} h}\right) \delta\left(p-p_{\mathrm{inj}}\right)
$$

where $\eta$ is the injection efficiency parameter, $n_{\mathrm{u}}$ is the plasma number density in the upstream region, $V_{\mathrm{s} h}$ is the shock speed, $V_{\text {wind }}$ is the wind velocity upstream of the shock, $R_{\mathrm{s} h}$ is the shock radius and $p_{\mathrm{i} n j}=\xi p_{\mathrm{th}}$ is the injection momentum, defined as a multiple of the momentum at the thermal peak of the Maxwellian distribution in the downstream plasma with temperature $T_{\mathrm{d}}$, $p_{\text {th }}=\sqrt{2 m k_{\mathrm{B}} T_{\mathrm{d}}}$. We assume the thermal leakage injection model [7], where $p_{\text {inj }}$ is the minimum momentum for which a thermal particle can cross the shock and enter the acceleration process and 


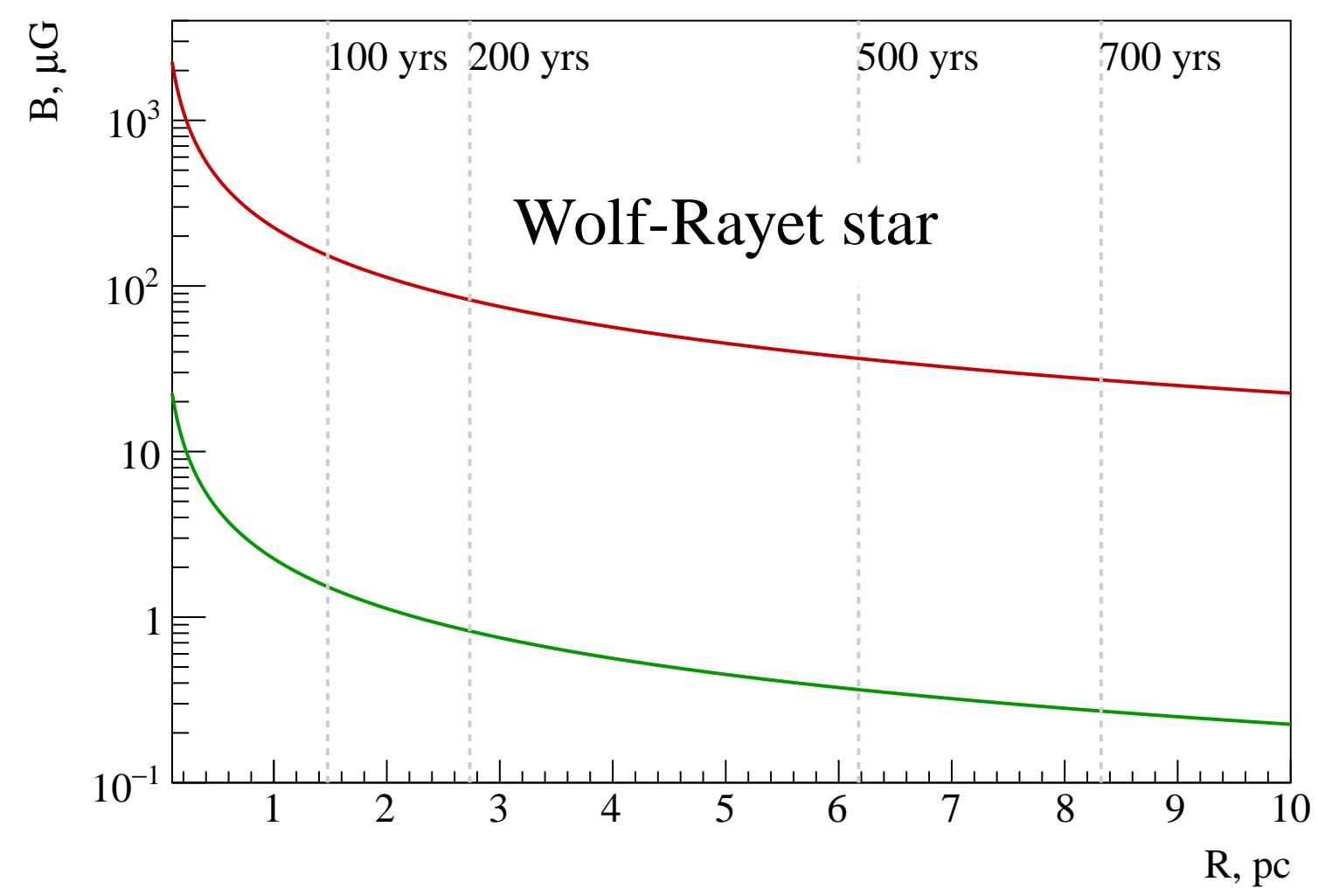

Figure 2: Same as Fig. 1 but for Wolf-Rayet star as a progenitor.

the injection efficiency for the compression ratio of 4 is determined as

$$
\eta=\frac{4}{\sqrt{\pi}} \frac{\xi^{3}}{e^{\xi^{2}}}
$$

We solve the transport equation for electrons in 1D using the RATPaC code as described in $[2,3,4,5]$ taking into account only a forward shock and ignoring a reverse shock. Resulting electron spectra at different moments of time are then used to simulate inverse Compton emission from the remnant. In this study we do not consider acceleration of protons and also assume that cosmic-rays will not dynamically effect the SNR evolution.

\section{Properties of the progenitor stars}

Red supergiants (RSGs) and Wolf-Rayet (WR) stars are known to be the most common progenitors of core-collapse SNRs. Stellar winds of these types of stars generate large stellar wind bubbles into which eventually expand SNRs. Their physical properties are however quite different which results in a different circumstellar environment.

\subsection{Red supergiants}

Initial masses of RSGs range from $8 M_{\odot}$ to $25 M_{\odot}$ (see e.g. [8]). During the RSG phase stars become very luminous and undergo strong mass-loss with the rate, $\dot{M}$, typically ranging from 
$\sim 10^{-7} M_{\odot} /$ yr to $\sim 10^{-5} M_{\odot} /$ yr [8]) and in some cases up to $10^{-4} M_{\odot} /$ yr [9]. The stellar wind is characterized by quite low velocity of $\sim 15$ to $\sim 50 \mathrm{~km} / \mathrm{s}$ [9]. In our hydrodynamic simulations of the RSG evolution we assume $\dot{M}=\sim 10^{-6} M_{\odot} / \mathrm{yr}$ and wind velocity $v_{\mathrm{w}}=20 \mathrm{~km} / \mathrm{s}$. The mass of the ejecta is assumed to be $3 M_{\odot}$

Following our parametrization (see Eq. 2.7) the circumstellar magnetic field is determined by the surface magnetic field of the star and stellar radius. RSGs exhibit relatively low surface magnetic fields of $1-10 \mathrm{G}[10]$ but a very large size of $\sim 100 R_{\odot}$ to $\sim 1000 R_{\odot}$. In this study we construct two test cases with stronger ( $\mathrm{cool}$ ) and weaker (not cool) effect on the resulting particle spectrum spectrum caused by synchrotron cooling. In the cool scenario we assume $B_{*}=10 \mathrm{G}$ and $R_{*}=1000 R_{\odot}$ and in the not cool scenario we set these parameters to $B_{*}=10 \mathrm{G}$ and $R_{*}=100 R_{\odot}$. in both cases we take an upper limit on the surface magnetic field as for lower magnetic field strength of $\sim 1 \mathrm{G}$ the effect becomes negligible even for large sizes of the star.

\subsection{Wolf-Rayet stars}

Wolf-Rayet stars have typical masses of $10-25 M_{\odot}$ but can be as heavy as $80 M_{\odot}$ in some cases [11]. WR stars feature similar mass-loss to RSGs with the $\dot{M}$ ranging from $5 \times 10^{-6} M_{\odot} / \mathrm{yr}$ to $5 \times 10^{-5} M_{\odot} / \mathrm{yr}$, but the stellar wind is much faster with wind velocity ranging from 1000 to 4000 $\mathrm{km} / \mathrm{s}$ [11]. In hydrodynamic simulations presented here we assume $\dot{M}=\sim 10^{-5} M_{\odot} / \mathrm{yr}$ and wind velocity $v_{\mathrm{w}}=2000 \mathrm{~km} / \mathrm{s}$. The mass of the ejecta is assumed to be $3 M_{\odot}$ same as for RSGs.

Wolf-Rayet stars are argued to be strongly magnetic with surface magnetic field of up to $1000 \mathrm{G}$, because the final product of their evolution are highly magnetized pulsars or magnetars with typical field of $10^{12}-10^{15} \mathrm{G}$ [11]. There are no direct measurements of the surface magnetic field, there is some evidence that magnetic field in some parts the wind can reach $100 \mathrm{G}$ implying surface magnetic field of $\sim 1000 \mathrm{G}$ [12]. These stars are also quite compact with a radius of only $1-10 R_{\odot}$ [11]. Based on the range of possible values we construct two cases: $\mathrm{cool}$ with $B_{*}=1000 \mathrm{G}$ and $R_{*}=10 R_{\odot}$ and not cool with $B_{*}=100 \mathrm{G}$ and $R_{*}=1 R_{\odot}$.

\section{Results}

Figures 3 and 4 show the inverse Compton emission generated by electrons accelerated at the shock of the remnant scattered on the CMB radiation field for RSG and WR star progenitors respectively. It can be seen that in the cool scenario the strong magnetic field encountered at early stages of the SNR evolution implies substantial synchrotron cooling which considerably modifies the electron spectrum and thus leaves a characteristic imprint in the spectrum of the gamma-ray emission. This characteristic feature shows up in the gamma-ray spectrum as a break at $\mathrm{GeV}$ energies, similar to the energy where a pion-decay signature is expected in the hadronic scenario. Above the break energy the gamma-ray spectrum softens resulting in a similar spectral shape to the gamma-ray emission produced in hadronic interactions. In general the resulting spectrum resembles the shape of the gamma-ray spectrum produced in the hadronic scenario creating new difficulties in discrimination between the leptonic and hadronic scenarios for the case when this cooling effect is strong enough.

This feature, however, gets smeared at later stages of evolution as the circumstellar magnetic field becomes weaker. Indeed, the lower ambient magnetic field is not strong enough anymore 
to cause severe synchrotron losses but still sufficient to accelerate particles to very high energies, which results in much more gamma-ray emission produced at later stages.

In the not cool scenario the impact of the synchrotron cooling on the resulting spectrum is almost negligible. This indicates that this effect strongly depends on the stellar parameters and therefore again demonstrates the importance of understanding the nature of the supernova progenitor for accurate modeling of the gamma-ray emission from SNRs.

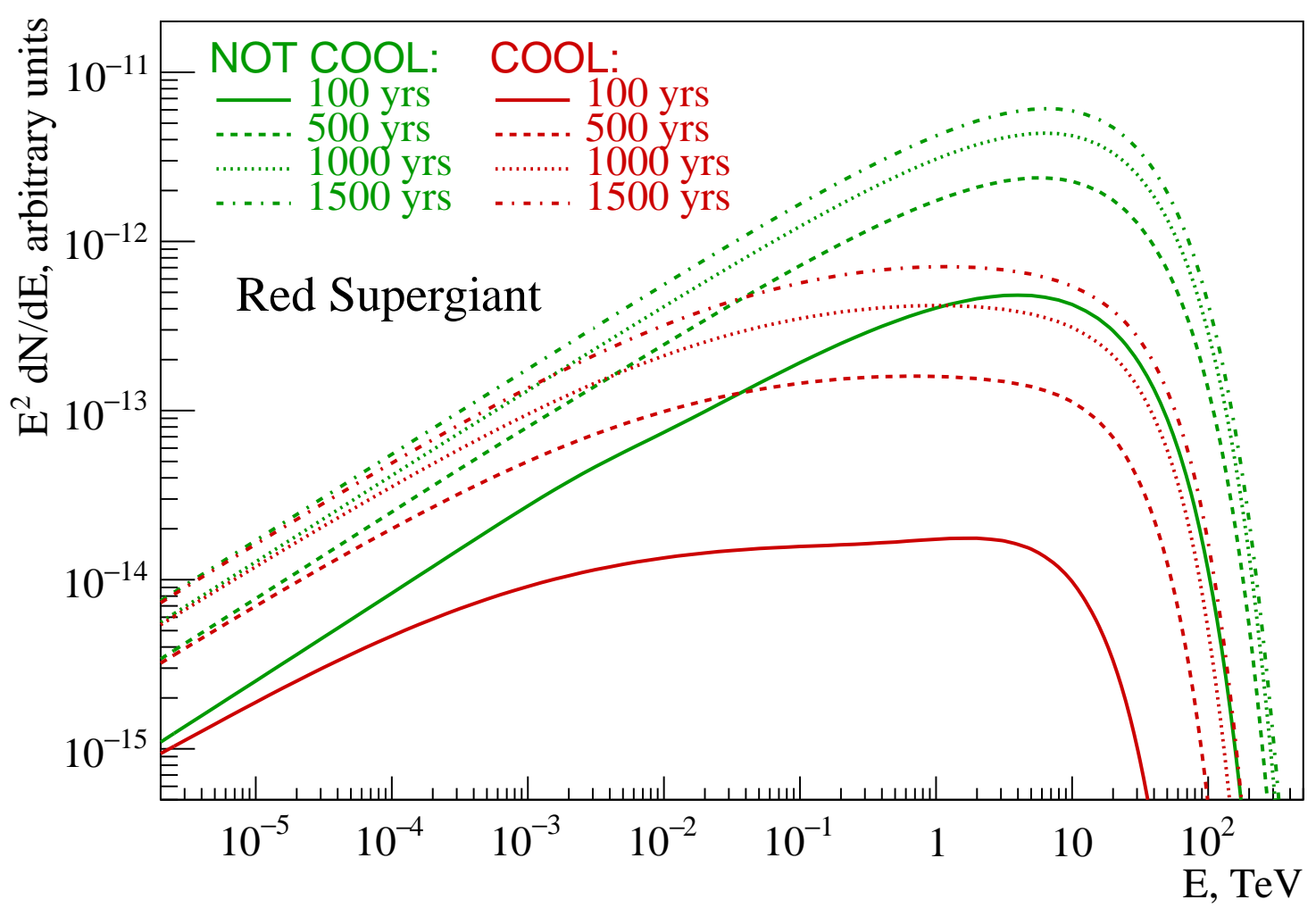

Figure 3: Inverse Compton emission from the SNR with a RSG progenitor for cool (red lines) and not cool (green lines) scenarios. Different line styles correspond to different ages of the remnant.

\section{Discussion}

The not cool scenario for the WR star shows the cut-off energy about an order of magnitude lower than in all other cases (Fig. 4). This is because the ambient magnetic field is also the lowest among all the considered cases (see Figs. 1,2) and not strong enough to contain particles close to the shock for efficient acceleration to high energies. The maximum electron energy is determined by the magnetic field far upstream. On the other hand synchrotron cooling is determined by the magnetic field downstream. It should be noted here again that in this study we ignored amplification of the magnetic field which might considerably change the picture. Indeed, if the circumstellar magnetic field gets strongly amplified in the immediate upstream of the shock and then compressed and transported downstream the synchrotron cooling effect would be stronger while the maximum electron energy would still be suppressed by low ambient field. In this case potentially the cooling 


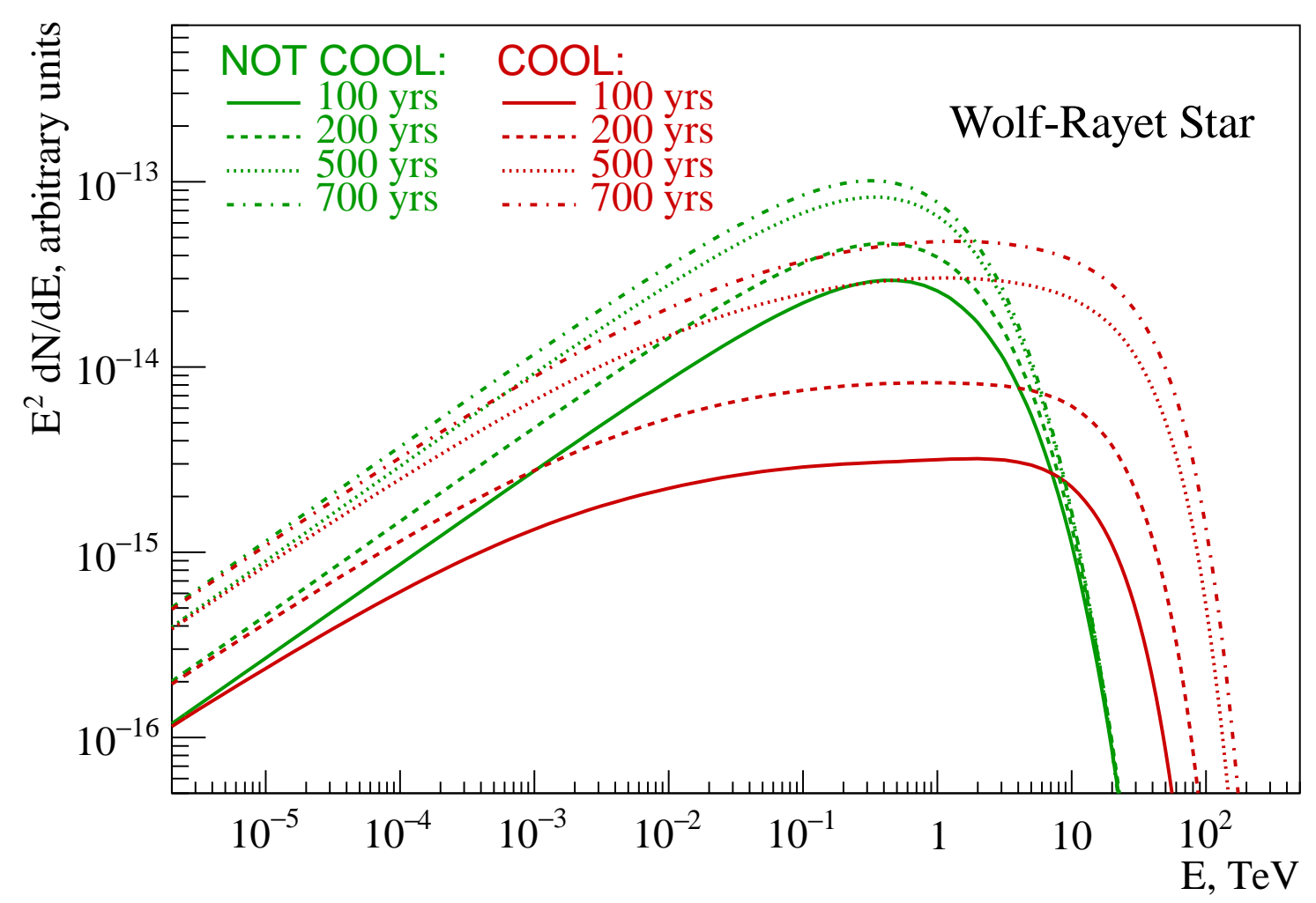

Figure 4: Same as Fig. 3 but with a Wolf-Rayet star as a progenitor.

feature at $\mathrm{GeV}$ energies would not get smeared with time but will remain in the spectrum even for very old remnants.

Another important factor which might have a strong impact on the spectral shape of the gamma-ray emission is the size of the stellar wind bubble. If the bubble is small enough to allow the remnant to reach its boundary and continue expanding into the interstellar medium, this might farther modify the electron and hence gamma-ray spectrum. Indeed, if the interstellar medium magnetic field is stronger than the one inside the bubble then outside the bubble electrons might start to accelerate to higher energies again introducing another kink in the gamma-ray spectrum. On the other hand, outside the bubble the shock would rapidly decelerate moving into a much denser environment and thus making acceleration less efficient in a way balancing the effect caused by the increased magnetic field. It is unclear which effect is going to have stronger impact and therefore this matter has to be farther investigated.

These and other issues which may arise in the course of the study will be thoroughly discussed in the paper which is currently under preparation.

\section{References}

[1] M. Ackermann, et al. Detection of the Characteristic Pion-Decay Signature in Supernova Remnants. Science, 339:807-811, February 2013. 
[2] I. Telezhinsky, V. V. Dwarkadas, and M. Pohl. Particle spectra from acceleration at forward and reverse shocks of young Type Ia Supernova Remnants. Astroparticle Physics, 35:300311, January 2012.

[3] I. Telezhinsky, V. V. Dwarkadas, and M. Pohl. Acceleration of cosmic rays by young corecollapse supernova remnants. AEAA, 552:A102, April 2013.

[4] Iurii Sushch, Robert Brose, and Martin Pohl. Modeling of the spatially resolved nonthermal emission from the Vela Jr. supernova remnant. AEAA, 618:A155, Oct 2018.

[5] R. Brose, I. Sushch, M. Pohl, K. J. Luken, M. D. Filipovic, and R. Lin. Non-thermal emission from the reverse shock of the youngest galactic Supernova remnant G1.9+0.3. arXiv e-prints, page arXiv:1906.02725, Jun 2019.

[6] O. Petruk, T. Kuzyo, and V. Beshley. Post-adiabatic supernova remnants in an interstellar magnetic field: parallel and perpendicular shocks. MNRAS, 456:2343-2353, March 2016.

[7] P. Blasi, S. Gabici, and G. Vannoni. On the role of injection in kinetic approaches to nonlinear particle acceleration at non-relativistic shock waves. MNRAS, 361:907-918, August 2005.

[8] Emma R. Beasor and Ben Davies. The evolution of red supergiant mass-loss rates. MNRAS, 475(1):55-62, Mar 2018.

[9] N. Mauron and E. Josselin. The mass-loss rates of red supergiants and the de Jager prescription. AEA A, 526:A156, Feb 2011.

[10] B. Tessore, A. Lèbre, J. Morin, P. Mathias, E. Josselin, and M. Aurière. Measuring surface magnetic fields of red supergiant stars. AEAA, 603:A129, July 2017.

[11] Paul A. Crowther. Physical Properties of Wolf-Rayet Stars. ARAEA, 45(1):177-219, Sep 2007.

[12] A. de la Chevrotière, N. St-Louis, A. F. J. Moffat, and MiMeS Collaboration. Searching for Magnetic Fields in 11 Wolf-Rayet Stars: Analysis of Circular Polarization Measurements from ESPaDOnS. ApJ, 781:73, February 2014. 